\title{
New Media
}

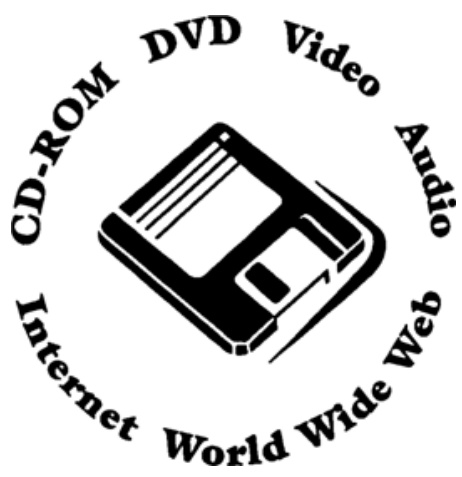

\section{Review of IARS 1998 Review Course Lectures CD- ROM}

This Windows / Mac CD-ROM produced by Thomas P. Engel MD and David A. Gilsdorf is sent free of charge to members of the International Anesthesia Research Society (IARS, http://www.iars.org). It contains audio recordings with slides and explanatory text from seven of the 1998 IARS Review Course Lectures presented at the 72nd Clinical and Scientific Congress of the International Anesthesia Research Society held at the Orlando World Center Marriott, Orlando, Florida, March 7-11, 1998. The date of the CD-ROM production is October 1998.

The presentations offered are:

- Modern Anesthetic Strategies for the Patient with Heart Disease (M. K. Cahalan)

- Preoperative Cardiac Evaluation Before Noncardiac Surgery (L. A. Fleisher)

- New Techniques for Labor Analgesia (J. L. Hawkins)

- Treatment of Shock - 1998 (R. G. Pearl)

- Perioperative Management of Head Trauma (D. S. Prough)

- Innovations in Ophthalmic Anesthesia (S. W. Stead)

- Pacemakers (J. R. Zaidan)

Included with the CD-ROM is a series of 28 multiple choice questions (four for each lecture) that can be completed for formal CME credit (no cost to IARS members).

I was able to install the software without trouble. I found the user interface very easy to use. The audio and slides were of good quality while the quality of the lecturers was consistently outstanding. Overall, I was quite pleased with the CD-ROM and recommend it without hesitation.

\section{Review of the ASA 1998 Annual Refresher Course Lectures CD-ROM}

This Windows / Mac CD-ROM contains the lecture outline notes for 99 of the Annual Refresher Course Lectures presented in October 1998 at the Annual Meeting of the American Society of Anesthesiologists (ASA) in Orlando, Florida. All the lecture notes are in PDF (Adobe Acrobat) format, although no multimedia capability other than support for graphic images is provided. The required Acrobat reader is included for those individuals without it.

I had no trouble installing and using the CD-ROM. The articles presented were well laid out and easy to read, although some of the equations were badly rendered. Printing individual articles was straightforward. Almost all articles are seven pages in length, making them especially appropriate for quick review. The CDROM also offers searching capability and information on educational objectives for each lecture. Unfortunately, there is no accompanying formal CME program with multiple choice questions.

The CD-ROM is available from the ASA (http://www.asahq.org) for \$15 USD plus shipping charges. It is excellent value at many times the price.

\section{Security on the internet}

Introduction to the issues

The last few years have seen enormous strides in providing Internet Security (IS) to the extent that IS technology is now available to support electronic commerce and other security-sensitive applications

Address correspondence to: Dr. D. John Doyle MD PhD FRCPC; New Media Editor, Canadian Journal of Anesthesia, Department of Anesthesia, The Toronto Hospital, 200 Elizabeth Street, Toronto, Ontario Canada M5G 2C4 Voice pager: (416) 375-0565, Fax: (416) 423-0452, e-mail: djdoyle@inforamp.net 
like banking or medical records transmission. With the latest security initiatives available from a host of vendors, secure communication and data transmission over the Internet is a reality today; IS is now primarily a matter of knowing what software to use. With modern IS technology and practices, your sensitive data such as credit cards numbers can be maintained in secret against almost all "hackers" (except possibly if the CIA takes a special interest in your messages). This does not mean that using your VISA card on the Internet will always be free of fraud potential, only that even the CIA will have trouble getting your number by intercepting encrypted data streams traveling on the Internet.

Fraud can still occur, for instance, if the clerk who eventually receives your order keeps a copy of your VISA card information for later criminal use. No decryption software needed there!

So, should one really be concerned about Internet privacy? The answer is yes. The connection between you and any other point on the Internet can be routed through dozens of independent systems, any of which can easily be monitored by unsavory technical types. You should consider non-encrypted e-mail, web browsing, chatting, and any other Internet use about as private as yelling to someone across a crowded room.

Most security issues in using the Internet usually involve one or more of the following:

- Picking up a virus or other pathogen from the 'net' or via E-mail

- Unauthorized visit to private files by unauthorized persons

- Destruction of data or files by deleting or renaming

- Denial of service (such as by changing your password without your knowledge)

- Impersonation or fraud (for example, using your account numbers)

- Theft of goods or services (for example, using your VISA number)

The above problems can be dealt with by appropriate IS precautions. The main goals of Internet security are: 1 . ensure people who say they are $\mathrm{X}$ are indeed $\mathrm{X}$ (authentication), 2. ensure that only $\mathrm{X}$ can read this message (encryption), and 3. provide protection against receiving harmful things (e.g., firewall protection, virus detection and eradication).

Some aspects of IS also concern "freedom of speech" issues, such as sites that provide information on do-ityourself bomb construction. There is the expectation that someone will eventually be killed because of information provided via by the Internet. Already there have been reports of people injured from bombs made from plans posted on the Internet. Similarly, reports of illicit intellectual property distribution via the Internet raises appropriate legal concerns. More recently, concerns about stolen physical property possibly being sold via Internet auction houses have lead to calls for police agencies to be kept up-to-date on Internet issues. Legal issues regarding liability are of particular interest where damage awards are sought via court action.

Security options for Internet and computer systems will vary from no particular security options at all, to simple password protection systems that are fairly easily hacked - but good enough to protect against $99 \%$ or more of users, up to complex professional/military schemes that are simply uncrackable in any reasonable time. Microsoft Internet Explorer and Netscape Navigator both have many security options that can be selected by the user.

Common sense security precautions are also important. These include:

- Change your password occasionally. To help ensure that your password is safe, change it often and use a so-called "strong" password - one that includes both letters and numbers (like "air2way") which is harder for hackers to break.

- Do not share your password with anyone. Most e-mail administrators will not ask for your password. If you get a message from someone purporting to be from your ISP or e-mail provider asking for your password, do not respond.

Exercise caution with attachments, especially those from unknown sources.

- Don't respond to "spam" (junk e-mail). Responding to unsolicited e-mail only confirms that you have an active e-mail address and will open you up to further solicitation and scam. To help control spam, some ISPs and e-mail services provide members with "filters" for incoming mail. These can easily be set up to send certain messages (such as those that include particular words or addresses) directly to your online trash can.

Keep your browser and Internet software updated. Developers of browsers, such as Microsoft Internet Explorer and Netscape Navigator, provide periodic updates that deliver enhanced security features and bug fixes.

- Use common sense and be smart when you're on the Internet. Maintain a healthy dose of skepticism in anything you read. Use caution when revealing personal information, such as your home address and phone number.

- Remember the "Elevator Rule": Never say in email what you would not say in a crowded elevator. 
- Check your VISA / MC / AmEx statements for unauthorized charges.

- Always use a virus checker for material down loaded from the Internet. 